\title{
Detection and Identification of Monaural and Binaural Pitch Contours in Dyslexic Listeners
}

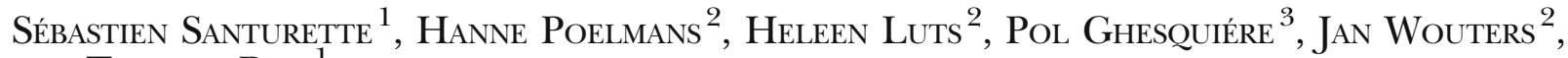 \\ AND TORSTEN DAU ${ }^{1}$ \\ ${ }^{1}$ Centre for Applied Hearing Research, Department of Electrical Engineering, Technical University of Denmark, DTU Bygning \\ 352, Ørsteds Plads, 2800, Kongens Lyngby, Denmark \\ ${ }^{2}$ Division of Experimental Otorhinolaryngology, Department of Neurosciences, Katholieke Universiteit Leuven, OEN2, \\ Herestraat 49 bus 721, 3000, Leuven, Belgium \\ ${ }^{3}$ Centre for Parenting, Child Welfare and Disabilities, Department of Educational Sciences, Katholieke Universiteit Leuven, \\ Andreas Vesaliusstraat 2 bus 3765, 3000, Leuven, Belgium
}

Received: 21 December 2009; Accepted: 9 March 2010; Online publication: 7 April 2010

\begin{abstract}
The use of binaural pitch stimuli to test for the presence of binaural auditory impairment in readingdisabled subjects has so far led to contradictory outcomes. While some studies found that a majority of dyslexic subjects was unable to perceive binaural pitch, others obtained a clear response of dyslexic listeners to Huggins' pitch (HP). The present study clarified whether impaired binaural pitch perception is found in dyslexia. Results from a pitch contour identification test, performed in 31 dyslexic listeners and 31 matched controls, clearly showed that dyslexics perceived HP as well as the controls. Both groups also showed comparable results with a similar-sounding, but monaurally detectable, pitch-evoking stimulus. However, nine of the dyslexic subjects were found to have difficulty identifying pitch contours both in the binaural and the monaural conditions. The ability of subjects to correctly identify pitch contours was found to be significantly correlated to measures of frequency discrimination. This correlation may be attributed to the similarity of the experimental tasks and probably
\end{abstract}

This research was in part financed by the fund for Scientific Research Flanders, grant G.0331.08, and a grant of the Research Council of Katholieke Universiteit Leuven, OT/07/034.

Correspondence to: Sébastien Santurette · Centre for Applied Hearing Research, Department of Electrical Engineering - Technical University of Denmark - DTU Bygning 352, Ørsteds Plads, 2800, Kongens Lyngby, Denmark. Telephone: +45-45253932; fax: +4545880577; email: ses@elektro.dtu.dk reflects impaired cognitive mechanisms related to auditory memory or auditory attention rather than impaired low-level auditory processing per se.

Keywords: dyslexia, binaural processing, pitch perception, frequency discrimination

\section{INTRODUCTION}

Developmental dyslexia is a specific learning impairment affecting the ability to fluently read, spell, and decode words, despite adequate educational opportunities and otherwise normal intellectual abilities (Lyon et al. 2003). The basis for this disorder, estimated to affect $5 \%$ to $10 \%$ of school-aged children (Shaywitz et al. 1990), is generally described by theories supporting two antagonistic points of view (see, e.g., Ramus 2003; Rosen 2003 for reviews). In short, the discussion revolves around whether the phonological processing disorder found in dyslexia is due to a purely cognitive deficit, linked to congenital dysfunction in the corresponding cortical areas of the brain, or to a basic sensorimotor deficit, possibly linked to a dysfunction of magno-cells along the sensory pathways. Conclusions favoring both points of view have been drawn from empirical data, and a possible relationship between low-level nonlinguistic impairment and reading disability remains under debate. 
Although their influence on reading abilities is controversial, a wide range of auditory processing disorders have been found in part of the dyslexic population (e.g., Wright et al. 2000; Amitay et al. 2002). Among other things, it has been suggested that low-level binaural processing might be impaired in some dyslexic listeners. McAnally and Stein (1996) obtained significantly lower binaural masking level differences (BMLDs) in their group of dyslexic listeners than in the control group, suggesting a difficulty of dyslexic listeners in exploiting interaural phase differences to obtain a binaural advantage. However, later studies (Hill et al. 1999; Amitay et al. 2002) found similar BMLDs in dyslexics and controls. Binaural pitch stimuli (Cramer and Huggins 1958) have also been used to investigate binaural processing abilities in dyslexic listeners. Dougherty et al. (1998) found that most of their dyslexic subjects failed at identifying and lateralizing pitch contours if no monaural cues were available. Their results suggested that the binaural integration of fine temporal information might be impaired in dyslexia, thus inducing an inability to perceive binaural pitch. Similarly, Edwards et al. (2004) asked a group of readingdisabled children to lateralize binaural pitch stimuli and found that $52 \%$ of dyslexics failed at the task in the absence of monaural cues. In contrast, Chait et al. (2007) did not find evidence for impaired binaural pitch perception in dyslexia. In a pitch-onset detection task, they compared the detectability of Huggins' pitch (HP) to that of sinusoidal targets in diotic noise (TN) and found that HP was generally perceivable by dyslexic listeners, with only few misses on average. Moreover, elevated response times with both HP and TN stimuli suggested a slower processing of pitchevoking noise stimuli in dyslexics, rather than an impairment in pitch detectability per se. Short stimulus durations or high task complexity might then have been responsible for the results obtained by Dougherty et al. (1998) and Edwards et al. (2004).

The question remains whether some readingdisabled listeners have impaired binaural pitch perception or whether all of them are able to hear binaural pitch, provided the duration of the stimuli is sufficiently long and the task simple enough. The present study aimed at clarifying this point, by investigating the ability of a larger group of dyslexic listeners to detect and identify binaurally and monaurally detectable pitch contours, using two different stimulus durations. By comparing the subjects' detection scores to their pitch contour identification scores and by evaluating correlations of the results with specific auditory and cognitive measures, the study aimed to verify or falsify the presence of low-level binaural processing impairment in dyslexia.

\section{METHODS}

\section{Procedure}

A pitch contour identification test was performed with two stimulus types, eliciting a pitch sensation in noise: a binaural pitch (BP) stimulus and a similarly sounding stimulus containing a monaurally detectable pitch (MP; see "Stimuli"). The use of two stimulus types was motivated by two factors: (a) Assuming that all listeners could perceive MP, it made pitch contour identification measureable in subjects unable to perceive BP; (b) It allowed evaluation of whether potential difficulties in pitch detection and contour identification were linked to a deficit in binaural processing or to a general difficulty in extracting tonal objects from background noise (c.f. Chait et al. 2007). The fundamental difference between these two stimuli is that while BP requires binaural presentation and cannot be perceived when listening with only one ear (in which case only noise is heard), MP can be detected monaurally.

In each trial, sequences of three musical notes were presented, such that they formed either rising, falling, or constant pitch contours (Table 1). Note frequencies were chosen to be between 500 and $800 \mathrm{~Hz}$, i.e., within the range of strongest salience of Huggins' pitch (Santurette and Dau 2007). The choice of rather large frequency intervals $(>17 \%)$ between successive notes in the rising and falling pitch contours (Table 1) was made to avoid possible effects of impaired frequency discrimination on pitch contour identification: France et al. (2002) obtained just noticeable differences (JNDs) that never exceeded

TABLE 1

Note frequencies, pitch contours, and frequency intervals used in the pitch contour identification experiment

\begin{tabular}{|c|c|c|c|c|c|}
\hline \multicolumn{2}{|c|}{ Note frequencies } & \multicolumn{2}{|c|}{ Pitch contours } & \multicolumn{2}{|c|}{ Frequency intervals } \\
\hline Note & Frequency $(\mathrm{Hz})$ & Contour & Note sequence & Notes & Interval \\
\hline $\mathrm{C}_{5}$ & 523.25 & Rising & $\mathrm{C}_{5}-\mathrm{E}_{5}-\mathrm{G}_{5}$ & $\mathrm{C}_{5}-\mathrm{E}_{5}$ & $136 \mathrm{~Hz}(23 \%)$ \\
\hline $\mathrm{E}_{5}$ & 659.26 & Falling & $\mathrm{G}_{5}-\mathrm{E}_{5}-\mathrm{C}_{5}$ & $\mathrm{E}_{5}-\mathrm{G}_{5}$ & $125 \mathrm{~Hz}(17 \%)$ \\
\hline $\mathrm{G}_{5}$ & 783.99 & Constant & $\mathrm{E}_{5}-\mathrm{E}_{5}-\mathrm{E}_{5}$ & & \\
\hline
\end{tabular}

Relative intervals between $f_{1}$ and $f_{2}$ are given in percent relative to $\left(f_{2}+f_{1}\right) / 2$ 
$16 \%$ of the test frequency $(500 \mathrm{~Hz})$ in their study of auditory frequency discrimination in dyslexia, and normal frequency sensitivity usually lies around 1-3\% in the frequency range considered here (Moore 2003). Two different note durations were used in order to measure whether performance in pitch detection and contour identification improved with stimulus duration: As response times obtained by Chait et al. (2007) were in the range $400-800 \mathrm{~ms}$ for both dyslexics and controls, note durations of $300 \mathrm{~ms}$ (shorter than the subjects' response time) and $900 \mathrm{~ms}$ (which should be long enough for all subjects to extract the pitches from the noise) were compared.

After each presentation, the subjects responded by pressing one of four buttons on a computer screen: an upward-pointing arrow (rising pitch), a downwardpointing arrow (falling pitch), a horizontal arrow (constant pitch), or a cross (no pitch). Subjects were instructed to press the cross when no melody was heard and to press the arrow corresponding to the perceived pitch contour when a melody was heard. The "no-melody" option was included so that both detection and contour recognition could be tested within a single short experiment. Subjects were presented 15 trials for each combination of stimulus type (MP or BP) and note duration (300 or $900 \mathrm{~ms}$ ). In addition to these 60 trials containing a pitch contour, 20 trials containing no pitch contour (diotic white noise only) were presented, half of them corresponding to each duration. This made it possible to evaluate false alarms and to avoid the possibility of subjects never pressing the cross. Trials were presented in a random order, and total testing time was approximately $10 \mathrm{~min}$ per subject. Before the test, each subject was first introduced to the different pitch contours played with pure-tone stimuli. A short 12trial practice run was also performed with pure tones to ensure that the task was correctly understood.

\section{Subjects}

Two groups of 31 dyslexic subjects (ages 19-30 years, mean 21.5) and 31 matched controls (ages 1932 years, mean 21.4) with normal hearing thresholds participated in the experiment. All experiments were approved by the Committee of Medical Ethics of Clinical Research of the Katholieke Universiteit Leuven. Subjects had Dutch as a native language and were matched according to gender, age, and educational level. The cognitive profiles of the two groups of subjects are summarized in Table 2. Dyslexics performed significantly worse than controls in all measures of reading and spelling accuracy, rapid automatized naming, phonemic awareness, and verbal working memory, despite scores similar to controls in measures of intellectual functioning. All dyslexics had nonword reading (Klepel test, van den Bos et al. 1994) scores below percentile 5 compared to the university norm group (Depessemier and Andries 2009) and had a formal diagnosis of developmental dyslexia. None of the controls reported any history of reading difficulties (Vandermosten et al. 2010). Psychoacoustic measures of temporal auditory processing were performed in the same two groups of subjects and are also included in Table 2, as they might shed light on the results. These measures included a tone-in-noise detection task, frequency modulation (FM) detection at a 2-Hz FM rate, and the JND in frequency at $490 \mathrm{~Hz}$. Details of the testing methods are given in Appendix 1.

\section{Stimuli}

Stimulus waveforms were generated in MATLAB $^{\circledR}$ with a 48,000-Hz sampling rate and 16-bit resolution in the following way: The BP stimulus was a Huggins' pitch and contained a frequency-dependent interaural phase difference pattern, such that the left and right noises were in phase at all frequencies, except for a narrow frequency range around the boundary frequency $f_{b}$. In the transition area around $f_{b}$, a phase difference varying linearly from 0 to $2 \pi$ was introduced in the frequency interval $\left[0.92 f_{b} ; 1.08 f_{b}\right]$ (see Fig. 1A), in order to create a pitch sensation corresponding to $f_{b}$. The stimuli were created as follows: (1) Random noise with the desired duration was generated in the spectral domain, using a $48,000-\mathrm{Hz}$ sampling rate; (2) All components of the noise were adjusted to have the same amplitude; (3) All frequency components above $4,000 \mathrm{~Hz}$ were set to zero; (4) The stimulus obtained in step 3 was kept intact, transformed back to the time domain using the inverse Fourier transform (iFFT) algorithm, and fed to the left channel; (5) Some of the phase components of the stimulus obtained in step 3 were modified in order to create the desired interaural phase difference pattern, and the iFFT algorithm was applied to the stimulus.

The MP stimulus was generated in the same way as the BP stimulus, except that no interaural phase difference was introduced, i.e., diotic broadband noise (BBN) was created. An additional diotic narrow band of noise (NBN) was then added to the BBN in the frequency interval $\left[0.96 f_{b} ; 1.04 f_{b}\right]$, thereby creating a pitch sensation corresponding to $f_{b}$, due to an increased amplitude of frequency components around $f_{b}$ (see Fig. 1B). In order to obtain a similar salience for the MP and BP stimuli, the overall level of the NBN was adjusted using a linear relationship with the overall level of the $\mathrm{BBN}$, following results from a preliminary salience adjustment experiment, described in Appendix 2. Although Huggins' pitch is 


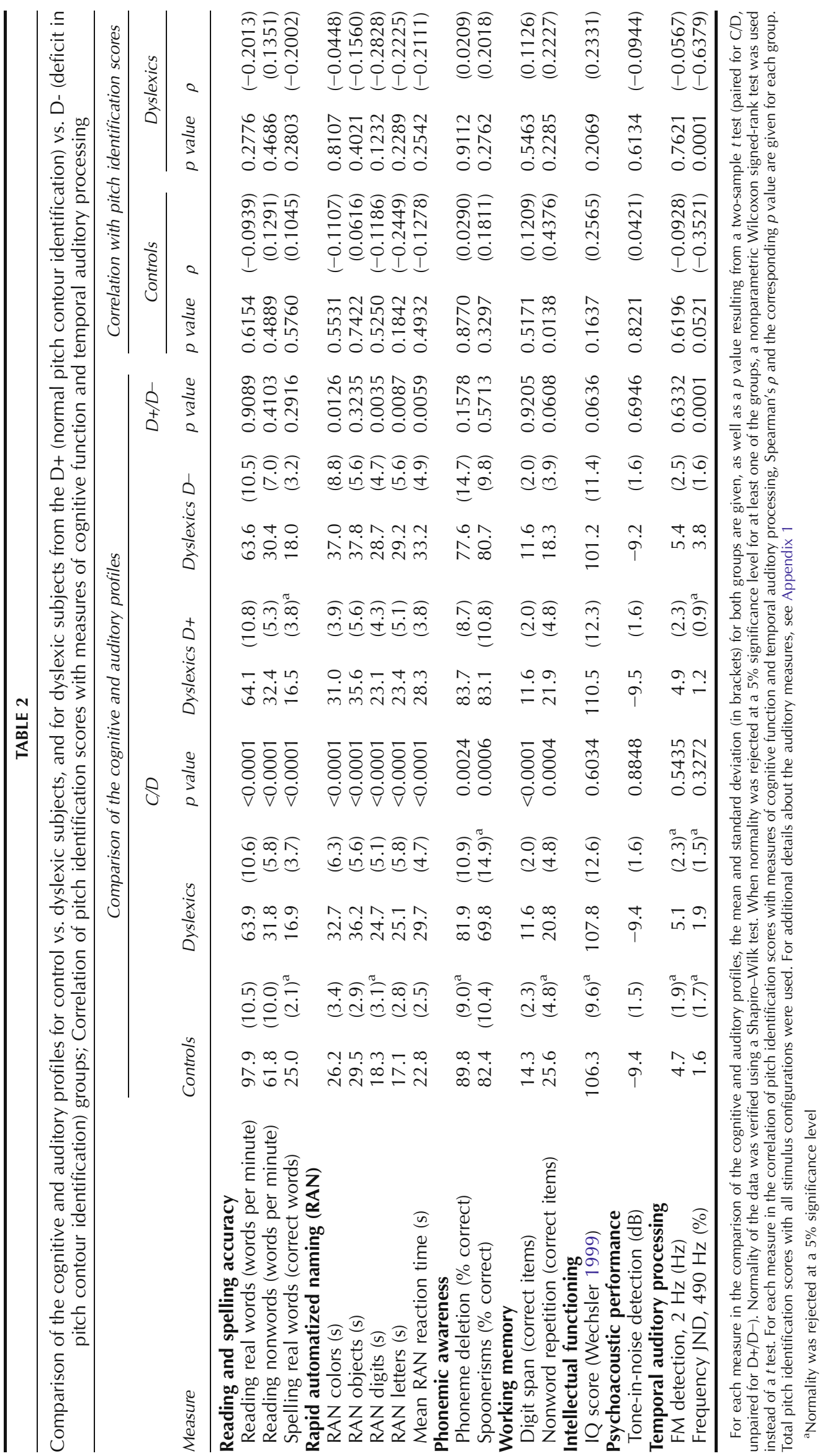



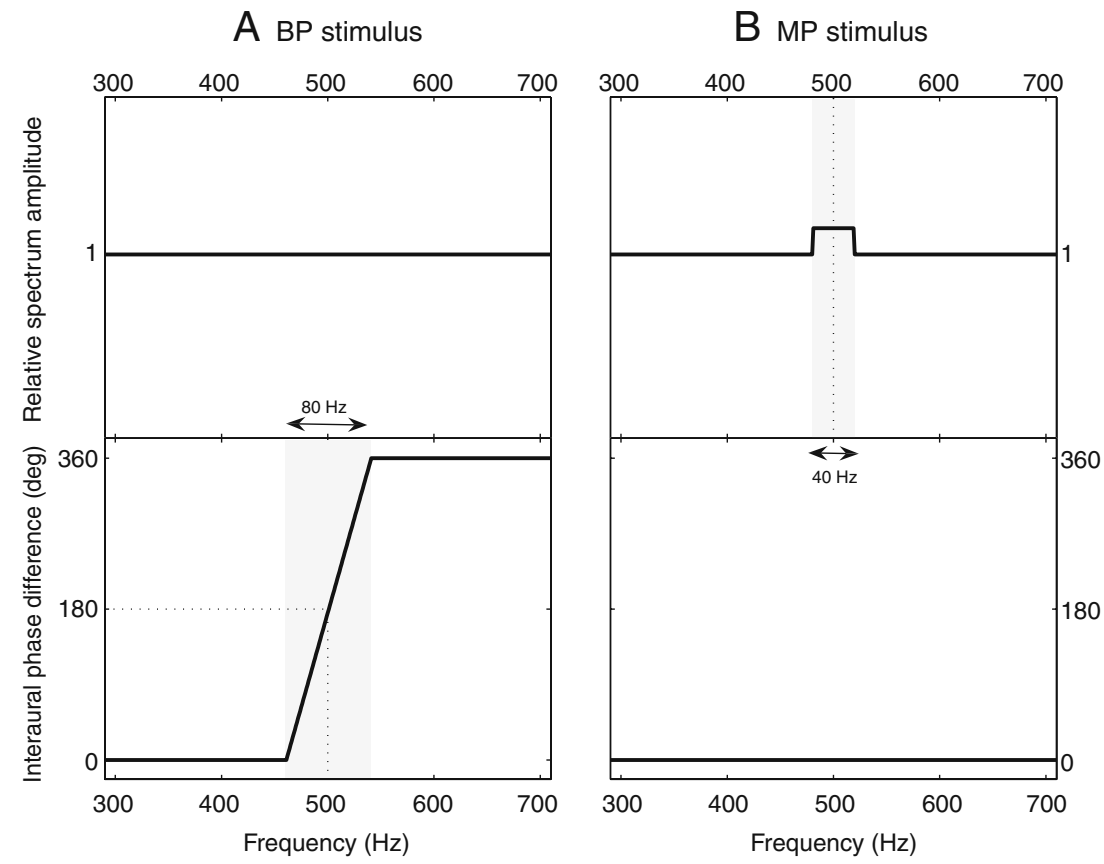

FIG. 1. Amplitude and phase spectra of the two stimuli used in the pitch contour identification experiment. A: binaural pitch (BP); B: monaurally detectable pitch (MP). Example for a boundary frequency $f_{b}=500 \mathrm{~Hz}$.

generally lateralized towards one side of the head, the BP configuration used in this experiment was previously found to have an ambiguous lateralization towards either the left or the right side of the head (c.f. $\mathrm{HP}^{-}$in Raatgever and Bilsen 1986). It was therefore chosen to introduce the NBN diotically rather than in one single channel, so that MP was perceived in the middle of the head.

For both stimulus types, each note was generated by adjusting $f_{b}$ to the desired note frequency. Notes were then concatenated to form the different pitch contours, and each contour was preceded and followed by $500 \mathrm{~ms}$ of diotic white noise (Fig. 2). In order to avoid discontinuities in the waveform between successive notes, 1-ms onset and offset cosine ramps were used at the beginning and end of each portion of the stimulus. The overall stimulus was gated with $100-\mathrm{ms}$ onset and offset cosine ramps. Wave files were created for each independent trial and implemented in the APEX 3 psychophysical platform (Francart et al. 2008). Stimuli were fed through a LynxONE soundcard and presented at an overall level of $70 \mathrm{~dB}$ SPL via Sennheiser HDA 200 headphones in a sound-attenuating listening booth. Subjects were not informed about the existence of different stimulus types.

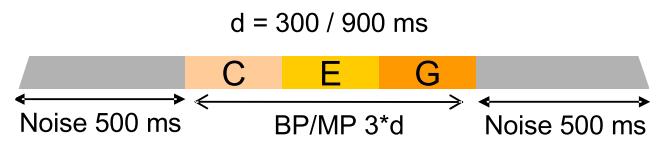

FIG. 2. Stimulus design for the pitch contour identification experiment. Example of a rising pitch contour.

\section{RESULTS}

\section{Pitch contour identification experiment}

Figure 3 shows the ability of control (light gray bars) and dyslexic (dark gray bars) subjects to detect the presence of pitch contours for each of the different stimulus configurations. It can be seen that subjects from both groups could clearly hear both MP and BP, independently of stimulus duration. In particular, the lowest overall score obtained among dyslexics with BP was $93 \%$, showing that all dyslexic listeners without

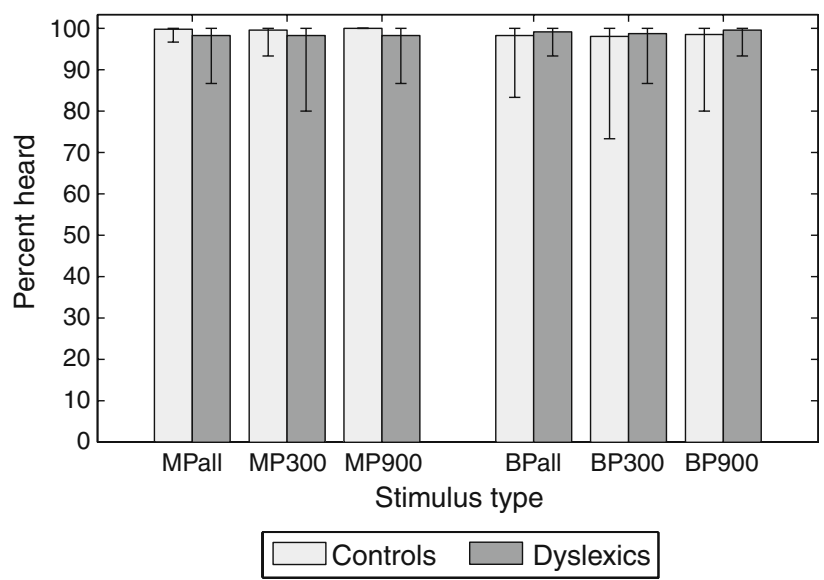

FIG. 3. Percentage of trials containing a pitch contour in which the pitch contour was detected (i.e., another button than the cross was pressed) for the different stimulus types. For MP and BP, the overall score regardless of duration is plotted first, followed by the scores for each duration independently (300 and 900 ms). Bar height corresponds to the mean over all subjects from a given group, and error bars indicate the lowest and highest scores among all subjects from that group. 
exception could hear binaural pitch. Differences between the two groups were overall not significant (MP300: $p=0.2344$, MP900: $p=0.0156$, BP300: $p=0.8594$, BP900: $p=0.3594$ [Wilcoxon signed-rank test]). N.B. None of the $p$ values mentioned in this article were corrected for multiple testing.

When comparing the total detection scores over trials with a $300-m s$ vs. a $900-m s$ note duration, no effect of stimulus duration was found on the ability of the listeners to detect the pitch contours (controls: $p=0.6719$, dyslexics: $p=0.4688$ [Wilcoxon signed-rank test]).

The average false alarm rate, i.e., the percentage of trials containing no pitch contour in which subjects pressed another button than the cross, was found to be rather low in both groups (dyslexics $8.5 \%$, controls $6.8 \%$ ) and never exceeded $35 \%$. This rules out the possibility that the high detection scores obtained here were due to strong false alarm bias or a misunderstanding of the task.

If one now considers the ability of subjects to correctly identify the pitch contours (Fig. 4), it appears that dyslexics are generally worse at the task than controls, in all stimulus configurations. This difference is only borderline significant when the whole group of dyslexic subjects is considered $(\mathrm{MP}+\mathrm{BP}$ overall identification score: $p=0.0402$ [Wilcoxon signed-rank test]). However, error bars in Figure 4 indicate that the variability among subjects is higher in dyslexics than in controls.

Figure 5A shows individual identification scores of $\mathrm{MP}$ and BP contours against each other. It can be seen that most dyslexic subjects actually performed similarly to controls (group D+, above the antidiagonal dashed line), while nine dyslexics (group $\mathrm{D}^{-}$) and one control identified less than $80 \%$ of pitch contours

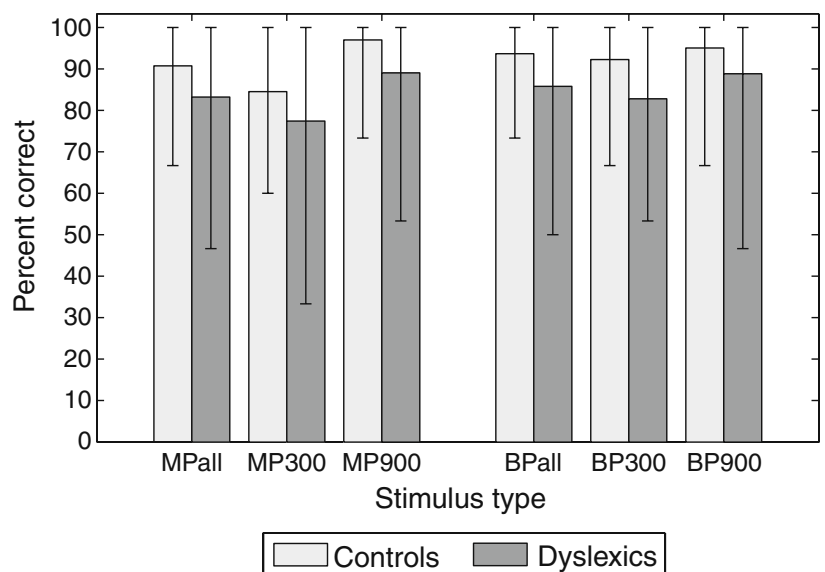

FIG. 4. Percentage of trials containing a pitch contour in which the pitch contour was correctly identified, for the different stimulus types. For MP and BP, the overall score regardless of duration is plotted first, followed by the scores for each duration independently (300 and $900 \mathrm{~ms}$ ). Bar height corresponds to the mean over all subjects from a given group, and error bars indicate the lowest and highest scores among all subjects from that group.

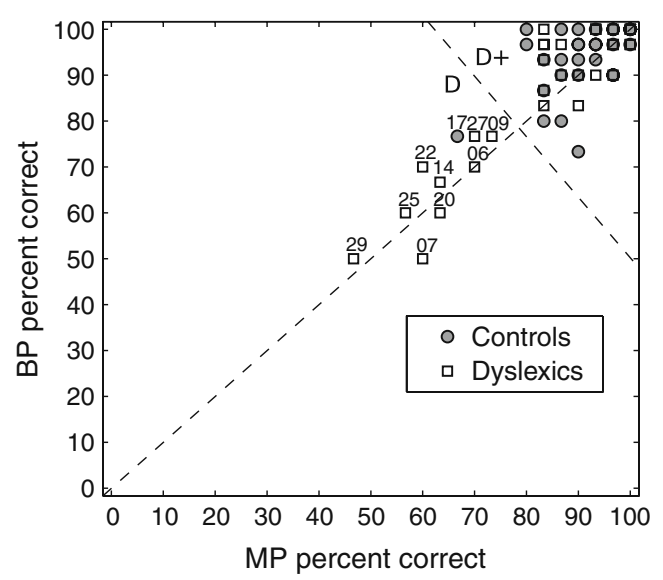

A Stimulus type

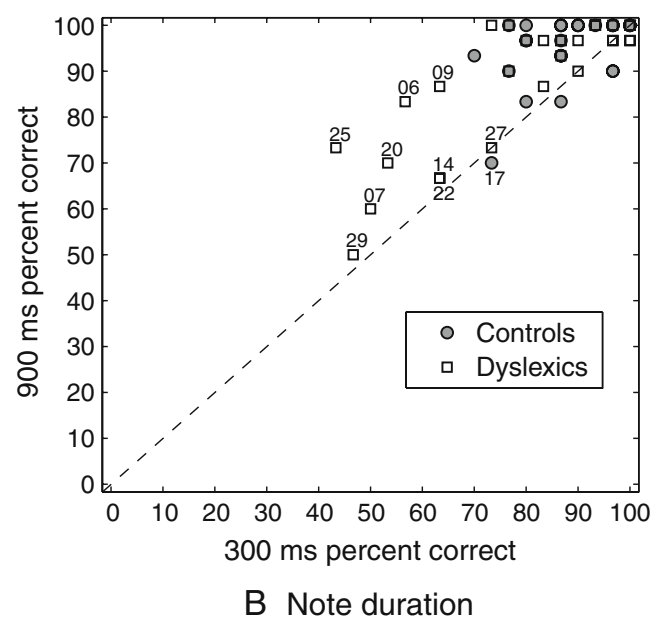

FIG. 5. Influence of stimulus type and note duration on the ability to correctly identify pitch contours: Percentage of trials containing a pitch contour in which the pitch contour was correctly identified: A with MP (horizontal axis) vs. BP (vertical axis) stimuli; B with 300-ms (horizontal axis) vs. 900-ms (vertical axis) note durations.

correctly with both MP and BP (points with number labels in Fig. 5A), thus indicating difficulty with the task. The fact that all data points lie around the diagonal line in Figure 5A reflects that the stimulus type did not have an influence on the task, i.e., the use of binaural pitch did not make pitch identification more difficult than for a monaurally detectable pitch. In fact, overall scores were on average higher with BP than MP. Moreover, seven of the ten labeled subjects in Figure 5A also obtained less than $80 \%$ correct identification in the practice run with pure-tone stimuli (only subjects 9, 14, and 27 obtained more than $80 \%$ correct in that condition), suggesting that their difficulty stems from the nature of the task rather than the type of stimulus used.

When comparing overall identification scores obtained with short vs. long note durations (Fig. 5B), it appears that almost all subjects benefited from a longer note duration (points generally fall above the 
diagonal line). Average scores for MP and BP stimuli were found to be significantly higher with $900-\mathrm{ms}$ notes than 300-ms notes in both groups of listeners (dyslexics: $p<0.0001$, controls: $p=0.0001$ [Wilcoxon signed-rank test]). The analysis of recorded reaction times revealed no significant difference between dyslexics and controls.

The analysis of error matrices showed that more misses occurred for the constant pitch contour than for the rising and falling pitch contours and that the rising and falling pitch contours were confused with each other more often than with the constant pitch contour. These trends were, however, similar in both the dyslexic and control groups.

\section{Correlation with measures of cognitive function} and temporal auditory processing

Overall, no significant correlations were found between pitch identification scores and cognitive measures included in the subjects' profile (Table 2), i.e., measures of reading and spelling accuracy, rapid automatized naming (RAN), phonemic awareness, working memory, and intellectual functioning. Taking multiple statistical testing into consideration, the only psychoacoustic measure that correlated significantly to performance in pitch contour identification was frequency discrimination in the dyslexic group. Performing the correlation analysis on the total pool of subjects (dyslexics and controls) led to even higher significance of the correlation between pitch contour identification scores and all measured frequency JNDs $(p<0.0001[\rho=-0.4960])$. A scatter plot of overall pitch contour identification scores vs. the measured frequency JNDs is given in Figure 6.



FIG. 6. Correlation between results in pitch contour identification and frequency discrimination. Total pitch identification scores with all stimulus configurations are plotted against the just noticeable difference (JND) in frequency at $490 \mathrm{~Hz}$. Straight lines show the correlation obtained over the whole pool of subjects (dashed line), and over the dyslexic group alone (dotted line).
When comparing the cognitive and auditory profiles of dyslexic subjects from groups $\mathrm{D}+$ and D- (see "Pitch contour identification experiment"), it appears that both groups show similar performance in most tasks (Table 2). However, a significant group difference was found for frequency discrimination abilities as well as RAN response times for digits, letters, colors, and mean RAN response times. It is also worth noting that the only control subject who had difficulty with pitch contour identification (subject 17) performed poorer than all other controls in several tasks (FM detection, spelling of real words) and obtained low scores in the phoneme deletion and digit span tests, despite similar reading scores to other controls. The latter subject also showed frequency JNDs that were overall considerably higher than in other controls.

\section{DISCUSSION}

The present results clearly show that dyslexic listeners are able to perceive binaural pitch. Moreover, the pitch detection scores of subjects with dyslexia were similar for the BP and MP stimuli. Because perceiving $\mathrm{BP}$ requires the comparison of accurate phase information across ears, these two findings suggest that no severe dysfunctions in peripheral temporal fine-structure processing or binaural integration mechanisms are associated with dyslexia and confirm the findings of Chait et al. (2007), who found no sign of binaural impairment in dyslexia using binaural pitch stimuli. This conclusion is supported by the fact that FM detection scores of the subjects with dyslexia suggested normal temporal fine-structure processing (Moore and Sek 1996) in dyslexic listeners. These normal FM detection scores contrast with the reduced FM detection abilities previously found in preschool children (Boets et al. 2007) and might reflect the presence of compensation mechanisms in these adult dyslexic subjects (university students).

The fact that all subjects could easily detect BP but that a subgroup of the dyslexics (29\%) had difficulty with pitch contour identification for both MP and BP stimuli suggests that the findings of Dougherty et al. (1998) and Edwards et al. (2004) may have been taskrelated: They used pitch contour identification and lateralization tasks, while Chait et al. (2007) used a simple detection task. Another difference that one should bear in mind is that the former two studies tested children, while the latter used adult subjects, who might have developed compensation mechanisms and thus show higher performance. However, it is unlikely that compensation can explain the whole of the present findings concerning binaural pitch perception: The presence of a subgroup with reduced 
performance in the present study confirms that the task remains problematic for these adult subjects. Moreover, because no influence of note duration on detection scores was found, it is unlikely that the results obtained by the former two studies were due to short stimulus durations. This is in line with findings from Banai and Ahissar (2006), who showed that the psychoacoustic abilities of dyslexic listeners with additional learning difficulties depended on the complexity of the required task rather than the nature of the presented stimuli. In the present study, the lack of significant difference between reaction times of dyslexics and controls may also reflect an influence of task complexity: While the present task required decision between four response buttons, leading to long response times in both groups of subjects, Chait et al. (2007) obtained significantly longer response times in dyslexics than in controls with a more automatic task.

One question raised by the present results concerns the origin of the difficulty of the $\mathrm{D}$ - group with pitch contour identification. Given the nature of the task and the experimental paradigm used in this study, several suggestions can be made that might explain this difficulty.

One explanation could be that dyslexics of the $\mathrm{D}^{-}$ group have a difficulty detecting tonal objects in background noise, as suggested by Chait et al. (2007). This would mean that, for these subjects, MP and BP are less salient than for the control and D+ groups, making pitch contour identification more difficult when using such stimuli. However, most Dsubjects also had difficulty with the task in the training session with pure tones, which contained no background noise. Additionally, no significant group difference between $\mathrm{D}+$ and $\mathrm{D}$ - subjects was found in the tone-in-noise detection task. Therefore, it is unlikely that a weaker pitch sensation with MP and BP stimuli was responsible for the lower pitch identification scores in the $\mathrm{D}$ - group.

Another explanation could be that, in the experimental procedure, subjects had to link an auditory pattern to a visual symbol: Each possible pitch contour corresponded to a different response button and, for instance, subjects had to link a rising pitch contour to an upward-pointing arrow. Such an ability might be impaired in some dyslexics. If such a deficit was the main reason for low pitch contour identification scores in the $\mathrm{D}^{-}$-group, one would expect $\mathrm{D}^{-}$- subjects to perform as $\mathrm{D}+$ subjects in a similar task that does not involve linking an auditory pattern to a visual symbol. In the present study, the frequency discrimination task was close to such a situation: While a comparison of the pitch of successive tones was required, no visual symbols had to be linked to specific pitch directions in the response process, as the subjects were instructed to identify the odd interval. However, a significant group difference in frequency JND was still found between $\mathrm{D}+$ and $\mathrm{D}^{-}$ subjects. This suggests that a difficulty linking auditory and visual patterns does not satisfactorily explain the results of the $\mathrm{D}^{-}$group.

Despite the rather large intervals between successive notes used in this study, one cannot exclude the possibility that impaired frequency discrimination was responsible for making pitch contour identification more difficult in the $\mathrm{D}^{-}$group. One might argue that the significant correlations between pitch contour identification scores and frequency JNDs support this hypothesis. However, frequency JNDs at $490 \mathrm{~Hz}$ in the D- group never exceeded $8 \%$ of the test frequency. Because frequency intervals in the pitch contours used here were larger than $17 \%$, it can be assumed that all subjects were able to discriminate between successive notes.

The question remains why some subjects failed to identify the individual contours even though they could hear the difference between them, and why frequency JNDs are then correlated to pitch contour identification scores. When comparing the subjects' tasks in the pitch contour identification and the frequency discrimination experiments, one can observe that they are, in fact, very similar: Frequency discrimination was measured using a three-alternative forced-choice (3AFC) paradigm, in which subjects listened to three successive tones before deciding which of these tones had a different pitch than the other two. This corresponds to choosing between three possible pitch contours and might explain why results from the two experiments strongly correlate.

This raises the question whether frequency discrimination per se is really impaired in some dyslexic listeners or whether the obtained results just reflect a limit imposed by a difficulty with the nature of the task itself. The present study used a three-interval, three-alternative forced-choice paradigm and found higher mean frequency JNDs in the dyslexic group than in the control group, but these group differences were not or only borderline significant. McAnally and Stein (1996) found a strongly significant difference between frequency JNDs of dyslexics and controls at $1 \mathrm{kHz}$, using a two-interval same-different paradigm in which the reference was presented once (2I-1A-X). Hill et al. (1999) measured frequency JNDs at 1 and $6 \mathrm{kHz}$ with a four-interval 2AFC paradigm in which the second or the third interval contained the target and found no group difference between dyslexics and controls at either test frequency. Considering such different results obtained using different tasks, it appears essential to investigate and discuss the influence of the experimental procedure on fre- 
quency JNDs with dyslexic listeners. In their comparison of thresholds obtained with a 2I-1A-X paradigm and a 2I-6A-X paradigm in which the reference was presented six successive times, France et al. (2002) showed that JNDs of dyslexic listeners could be reduced to those of controls by increasing the number of available observations and using short interstimulus intervals. They suggested that a deficit in early auditory memory (Hari et al. 1999) could explain the dependence of JNDs on the procedure used and argued that repeated exposure to known identical references might help stabilize auditory memory and thus lead to lower thresholds.

If the difficulty of $\mathrm{D}$ - subjects in identifying pitch contours disappears when changing the experimental procedure, this would confirm that these subjects are in fact able to perceive the difference between successive stimulus intervals and that their difficulties are directly linked to the nature of the task. Therefore, mechanisms responsible for the ability to retain successive stimulus intervals in memory could be deficient. This would be consistent with the presence of a significant $\mathrm{D}+/ \mathrm{D}-$ group difference in a discrimination task (frequency JND), but not in less-complex detection tasks (tone-innoise and FM detection). This hypothesis would also be in line with findings from Banai and Ahissar (2004): A subgroup of their dyslexic subjects (DP) obtained frequency JNDs that were elevated compared to other dyslexics, and subjects from this subgroup, which formed a proportion similar to that of the $\mathrm{D}$ - group of the present study, were also the ones showing a significant impairment in verbal working memory. Moreover, in both studies, significantly longer RAN reaction times were found in subjects from the aforementioned subgroups (DP in Banai and Ahissar (2004), D- in the present study), compared to other dyslexic subjects. This suggests that the core phonological deficit of $\mathrm{D}+$ and $\mathrm{D}-$ subjects might have different etiologies and that a deficit in rapid information retrieval from memory plays a role in the difficulty of $\mathrm{D}-$ subjects with the pitch contour identification and frequency discrimination tasks.

Finally, because pitch contour identification involves following changes in pitch, the ability to switch attention from one pitch percept to the next could also be impaired in the $\mathrm{D}^{-}$group. This would be consistent with findings from Hari and Renvall (2001) whose results suggested that "sluggish attentional shifting" could give rise to impaired processing of rapid stimulus sequences. More recently, Hämäläinen et al. (2008) measured event-related potentials (ERP) in reading-disabled children and found that ERP responses to pitch changes were lower in readingdisabled children than in control children, in a component related to attention switching.

\section{CONCLUSION}

It was found that binaural pitch was easily detectable in both dyslexic listeners and matched controls, which suggests intact low-level binaural processing in dyslexia. In both groups of subjects, pitch contour identification scores were similar for binaural pitch stimuli and monaurally detectable pitches in noise, showing no sign of low-level binaural impairment in dyslexic listeners. A subgroup of dyslexics showed difficulties with pitch contour identification. Results in that experiment were significantly correlated with measures of frequency discrimination, and this correlation is most likely due to the similarity of the tasks in the two experiments. The difficulty in such tasks is thought to be attributable to auditory memory or auditory attention deficits, rather than reduced frequency discrimination abilities per se. The results favor impaired cognitive mechanisms as precursors to reading disability, rather than impaired low-level auditory processing. They underline the influence of the choice of the experimental paradigm and the task of the subjects on results from basic psychophysical measures with dyslexic listeners. Overall, great care ought to be taken before asserting the presence of a low-level auditory processing deficit in a dyslexic group, if the task involves auditory memory or auditory attention to a nonnegligible extent.

\section{ACKNOWLEDGEMENTS}

The authors would like to thank two anonymous reviewers for their helpful comments on an earlier version of this manuscript.

\section{Appendix \\ Methods used for auditory processing measures}

A 3AFC procedure was used in all experiments. Psychoacoustic performance: Detection of a $1-\mathrm{kHz}$ pure tone in background noise was measured. Results are given as the signal-to-noise ratio at threshold in decibels. FM detection: Detection of frequency modulation of a $1-\mathrm{kHz}$ tone was measured for a $2-\mathrm{Hz} \mathrm{FM}$ rate. Results are given as the maximum frequency excursion at threshold in Hertz. Frequency discrimination: The just noticeable difference in frequency was measured at $490 \mathrm{~Hz}$ using a two-down one-up procedure and a fixed-reference paradigm. Targets were always lower in frequency than the reference tone, and the target frequency was varied by a factor of 1.4. Stimuli were presented monaurally at $70 \mathrm{~dB}$ SPL. Results are given as the smallest detectable change in frequency (in percent of the test frequency). 


\section{Salience adjustment of MP and BP}

In order to match the salience of MP to that of BP, a preliminary salience adjustment experiment was performed by five normal-hearing listeners. A 2I-2AFC procedure was used, in which one random interval contained a BP stimulus, and the other interval contained an MP stimulus. The overall level of the broadband noise in both MP and BP stimuli $\left(L_{\mathrm{BBN}}\right)$ was fixed, and the tracking variable was the overall level of the additional narrow band of noise in the MP stimulus $\left(L_{\mathrm{NBN}}\right)$. For each presentation, the task of the listener was to indicate, via a computer interface, in which interval the pitch was more salient. Intervals had a 500-ms duration, including 30-ms onset and offset cosine ramps, and were separated by a $500-\mathrm{ms}$ silent pause. A one-up one-down procedure was used: when BP was perceived as more salient, $L_{\mathrm{NBN}}$ was increased in the next presentation, and when MP was perceived as more salient, $L_{\mathrm{NBN}}$ was decreased in the next presentation. The starting value of $L_{\mathrm{NBN}}$ was $75 \mathrm{~dB}$ SPL. Step sizes of $8,4,2$, and $1 \mathrm{~dB}$ were used, and the step size was decreased after each upper reversal. A run was terminated after 14 reversals, and the threshold value was determined from all points following the sixth reversal. The experiment was performed for $L_{\mathrm{BBN}}=[55 ; 60$; $65 ; 70 ; 75 ; 80]$, with $f b=500 \mathrm{~Hz}$. Stimuli were generated as described in "Stimuli." Each subject performed three runs for each value of $L_{\mathrm{BBN}}$. The best-matching $L_{\mathrm{NBN}}$ value was defined as the average value obtained over all runs. The average $L_{\mathrm{NBN}}$ giving equal salience was found to be linearly correlated to $L_{\mathrm{BBN}}$, and a first-degree polynomial, described by $L_{\mathrm{NBN}}=1.07 \times L_{\mathrm{BBN}}-15.69$, was fitted to the data. This relationship was used to generate the MP stimulus for the pitch contour identification experiment (see "Stimuli").

\section{REFERENCES}

Amitay S, Ahissar M, Nelken I (2002) Auditory processing deficits in reading disabled adults. J Assoc Res Otolaryngol 3:302-320

Banai K, Ahissar M (2004) Poor frequency discrimination probes dyslexics with particularly impaired working memory. Audiol Neurootol 9:328-340

Banai K, Ahissar M (2006) Auditory processing deficits in dyslexia: task or stimulus related? Cereb Cortex 16:1718-1728

Boets B, Wouters J, van Wieringen A, Ghesquière P (2007) Auditory processing, speech perception and phonological ability in preschool children at high-risk for dyslexia: a longitudinal study of the auditory temporal processing theory. Neuropsychologia 45:1608-1620

Chait M, Eden G, Poeppel D, Simon JZ, Hill DF, Flowers DL (2007) Delayed detection of tonal targets in background noise in dyslexia. Brain Lang 102:80-90

Cramer EM, Huggins WH (1958) Creation of pitch through binaural interaction. J Acoust Soc Am 30(5):413-417

Depessemier P, Andries C (2009) Gletschr, test voor gevorderd lezen et schrijven. Garant, Antwerp
Dougherty RF, Cynader MS, Bjornson BH, Edgell D, Giaschi DE (1998) Dichotic pitch: a new stimulus distinguishes normal and dyslexic auditory function. NeuroReport 9(13):3001-3005

Edwards VT, Giaschi DE, Dougherty RF, Edgell D, Bjornson BH, Lyons C, Douglas RM (2004) Psychophysical indexes of temporal processing abnormalities in children with developmental dyslexia. Dev Neuropsychol 25(3):321-354

Francart T, van Wieringen A, Wouters J (2008) APEX 3: a multipurpose test platform for auditory Psychophysical Experiments. J Neurosci Meth 172:283-293

France SJ, Rosner BS, Hansen PC, Calvin C, Talcott JB, Richardson AJ, Stein JF (2002) Auditory frequency discrimination in adult developmental dyslexics. Percept Psychophys 64(2):169-179

Hämäläinen JA, Leppänen PHT, Guttorm TK, Lyytinen H (2008) Event-related potentials to pitch and rise time change in children with reading disabilities and typically reading children. Clin Neurophysiol 119:100-115

HaRi R, Renvall H (2001) Impaired processing of rapid stimulus sequences in dyslexia. Trends Cogn Sci 5(12):525-532

Hari R, SäÄskilahti A, Helenius P, Uutela K (1999) Non-impaired auditory phase locking in dyslexic adults. NeuroReport 10:23472348

Hill NI, Bailey PJ, Griffiths YM, Snowling MJ (1999) Frequency acuity and binaural masking release in dyslexic listeners. J Acoust Soc Am 106(6):L53-L58

Lyon GR, Shaywitz SE, ShaYwitz BA (2003) A definition of dyslexia. Ann Dyslexia 53:1-14

McAnally KI, Stein JF (1996) Auditory temporal coding in dyslexia. Proc R Soc Lond B 263:961-965

Moore BCJ (2003) An introduction to the psychology of hearing, 5 th edn. Academic, New York

Moore BCJ, Sek A (1996) Detection of frequency modulation at low modulation rates: evidence for a mechanism based on phase locking. J Acoust Soc Am 100(4, Pt. 1):2320-2331

RAATGEVER J, BiLSEN FA (1986) A central spectrum theory of binaural processing. Evidence from dichotic pitch. J Acoust Soc Am 80 (2):429-441

Ramus F (2003) Developmental dyslexia: specific phonological deficit or general sensorimotor dysfunction? Curr Opin Neurobiol 13:212-218

Rosen S (2003) Auditory processing in dyslexia and specific language impairment: Is there a deficit? What is its nature? Does it explain anything? J Phonetics 31:509-527

SANTURette S, DAU T (2007) Binaural pitch perception in normalhearing and hearing impaired listeners. Hear Res 223(1-2): $29-47$

Shaywitz SE, Shaywitz BA, Fletcher JM, Escobar MD (1990) Prevalence of reading disability in boys and girls. Results of the Connecticut longitudinal study. J Am Med Assoc 264(8):998-1002

van den Bos KP, Spelberg HCL, Scheepstra AJM, De VRies JR (1994) De Klepel. Vorm A en B. Een test voor de leesvaardigheid van pseudowoorden. Verantwoording, handleiding, diagnostiek en behandeling. Nijmegen, Berkhout

Vandermosten M, Boets B, Luts H, Poelmans H, Golestani N, Wouters J, GHESQuiEke P (2010) Adults with dyslexia are impaired in categorizing speech and non-speech sounds on the basis of temporal cues. P Natl Acad Sci USA (in press)

WechsLer D (1999) Wechsler adult intelligence scale, 3rd edn. The Psychological Corporation, London

Wright BA, Bowen RW, Zecker SG (2000) Nonlinguistic perceptual deficits associated with reading and language disorders. Curr Opin Neurobiol 10:482-486 